\title{
A double-Bragg detector with digital signal processing for the event-by-event study of fission in actinide nuclei
}

\author{
R.J.W. Frost \\ School of Physics and Astronomy, The University of Manchester, \\ Manchester, M13 9PL, England \\ robert.frost-2@manchetser.ac.uk \\ A.G. Smith \\ School of Physics and Astronomy, The University of Manchester, \\ Manchester, M13 9PL, England \\ gavin.smith@manchester.ac.uk
}

Published 1 September 2016

\begin{abstract}
In the current paper, a windowless double-Bragg chamber incorporating full signal digitisation has been developed for the purpose of studying the energy (E), mass (A), charge ( $Z$ ) and angular distributions $(\theta, \Phi)$ of nuclei generated by fission. This device measures $\mathrm{E}$ for each fission fragment by collection of the charge produced during ionisation of the fill gas. Subsequent digitisation of the signals from each of two anodes yields information on $\mathrm{dE} / \mathrm{dx}$, as well as electron collection time, which can be further used for polar angle $(\theta)$ determination. Frisch-grid and cathode signals are also digitised and are used both for anode signal correction and to produce further information on $\theta$. To verify the operation of this detector, three angular determination techniques from the literature were implemented, and the results were found to be consistent with the referenced paper. Current results from the spontaneous fission of ${ }^{252} \mathrm{Cf}$ are presented.
\end{abstract}

Keywords: Bragg detector; ionization chamber; fission.

\section{Introduction}

Since the 1960s, there have been many measurements of fission yield and fragment kinetic energy distributions for spontaneous and thermal neutron-induced fission of actinide nuclei. ${ }^{1}$ Of the event-by-event measurement techniques available, mass determination by the $2 \mathrm{E}$ method, ${ }^{2}$ where the energies of both fragments are recorded independently, offers an advantage over $2 \mathrm{v}, \mathrm{Ev}$ and mass separation, in that it can be performed using very close geometry detectors, the large solid angle facilitating the use of low fission rate targets without a dramatic increase in beam flux or a damaging increase in target thickness. 2E devices such as the double-Bragg detector have experienced somewhat of a renaissance over the past decade, as advances in digital signal processing (DSP) technology have dramatically improved both the quality and quantity

This is an Open Access article published by World Scientific Publishing Company. It is distributed under the terms of the Creative Commons Attribution 3.0 (CC-BY) License. Further distribution of this work is permitted, provided the original work is properly cited. 
of the data they are able to produce. In the current paper, a double gridded-Bragg detector (DGBD) has been designed and commissioned; its performance has been tested using modern angular determination techniques.

\section{Principles of Detector Operation}

A schematic diagram of the detector is presented in Fig. 1. The placement of the source is such that a large solid angle is covered by each side of the detector, regions designated: source-side (SS) and backing-side (BS). Each of the fragments generated in a fission event will, in general, enter a different region of the detector. The charge liberated as a fragment stops in the fill gas and drifts toward its respective grid with constant velocity in the longitudinal electric field. Upon passing the grid, a step change in electric field strength is experienced and the charge is rapidly collected by the anode.

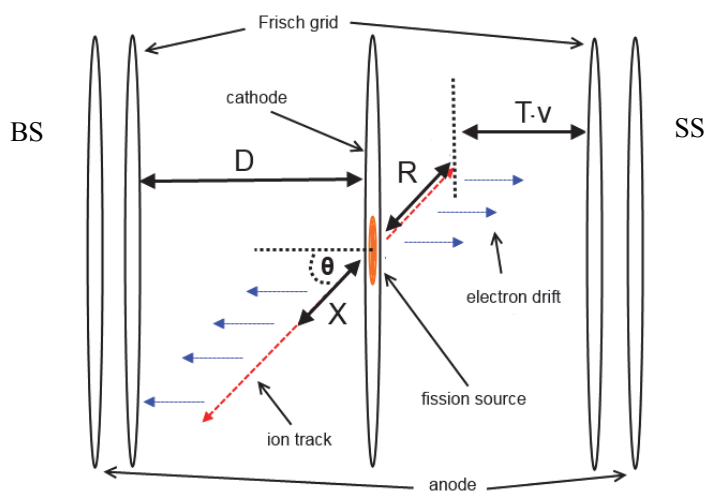

Fig. 1. Schematic diagram of the double gridded-Bragg detector.

As the charge moves across the drift region (DR), between the cathode and grid, it will induce an instantaneous current in the surrounding electrodes according to the RamoShockley theorem: ${ }^{3}$

$$
\boldsymbol{i}=\boldsymbol{E q v}
$$

where $\mathrm{q}$ is the charge in motion, $\mathrm{v}$ its velocity and $\mathrm{E}$ is the electric field in the direction of $\mathrm{v}$, under the conditions that the charge is not present, the electrode in question is at unit potential and all others are grounded.

The instantaneous current introduces a distortion into the desired output signal related to the initial position of the charge. The grid serves to separate the position and magnitude components of the output signals, the grid taking the former and the anode the latter, although the shielding the grid gives to the anode cannot be perfect and later correction is still required. The grid inefficiency (GI) can be determined either analytically ${ }^{4}$ or experimentally. ${ }^{5}$ 
The data acquisition system is triggered from the cathode output signal; anode, grid and cathode outputs are all digitized. The full height of the anode signal is proportional to the energy deposited by the fission fragment (E) and its derivative to the fragments stopping-power (SP) coupled with a polar-angle related component. Example signal traces are presented in Fig. 2.

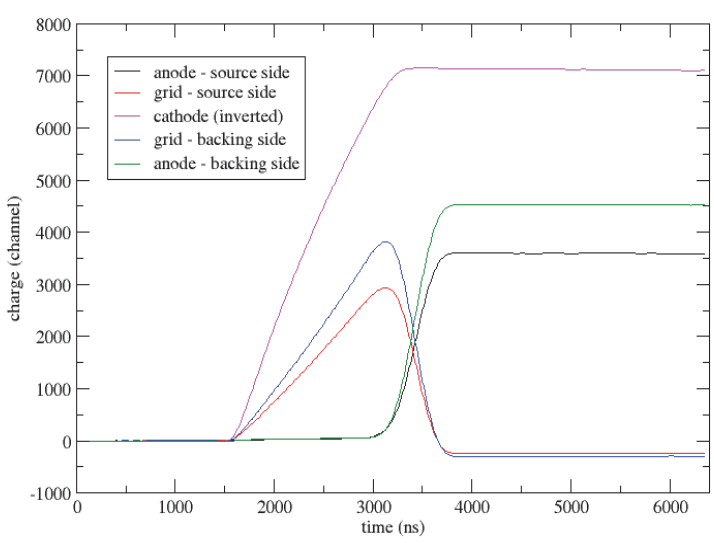

Fig. 2. Example signal traces from the double-Bragg detector.

By application of Eq. (1), it can be shown that fragment emission angle is related to grid signal height ${ }^{6}$ by:

$$
\cos (\boldsymbol{\theta})=\frac{Q_{G}^{\operatorname{corr}}}{Q_{A}^{\operatorname{corr}}\left(\frac{\bar{X}}{D}\right)},
$$

where $Q_{A}^{\text {corr }}$ and $Q_{G}^{\text {corr }}$ are the charge on the anode and grid after GI correction and $\bar{X} / D$ is the ratio of the distance between the origin and the center of mass of the electron cloud before the drift to the cathode-grid distance. This is analogous to the relation of drift time to fragment emission angle: ${ }^{7}$

$$
\cos (\theta)=\frac{\mathrm{D}-\mathrm{vT}}{\mathrm{R}},
$$

where $\mathrm{T}$ is the time between the fission event and the first electrons reaching the grid, $\mathrm{v}$ is the drift velocity, and $\mathrm{R}$ is the length of the ionization track; the expression (D-Tv) is the collection time. Equations (2) and (3) form distributions in each energy channel that can be fitted to obtain $\theta$. With $\theta$ determined, angular dependent corrections to $\mathrm{E}$ and SP can be made. From the corrected energies, the mass (A) of the fragments can be determined by applying conservation of momentum:

$$
\boldsymbol{A}_{\mathbf{1}}=\frac{E_{2} A_{p}}{E_{1}+E_{2}}
$$


where $A_{1}$ is the mass of the SS fragment, $A_{p}$ is the mass of the parent nuclei and $E_{1}$ and $\mathrm{E}_{2}$ are the energies of the SS and BS fragments. All data corrections and analyses are performed using off-line DSP techniques.

\section{Experimental Setup}

All developmental work and data collection was performed at the University of Manchester. The diameter of the chamber's drift region is $14 \mathrm{~cm}$, cathode-grid distance is $7 \mathrm{~cm}$ with field shaping rings every $1 \mathrm{~cm}$, and grid-anode distance is $1 \mathrm{~cm}$. The grid is made from a fine mesh of $90 \%$ physical transparency, clamped between two $1 \mathrm{~mm}$ thick copper rings. The anode is made from a $1 \mathrm{~mm}$ thick copper disk and the cathode from a $0.5 \mathrm{~mm}$ copper disk with a central, $2 \mathrm{~cm}$ cutout for the source holder. The detector housing is made from $5 \mathrm{~mm}$ thick aluminum.

The source is made by liquid deposition and then evaporation of a concentrated $\mathrm{HCl}$, ${ }^{252} \mathrm{Cf}$ solution onto a $0.5 \mu \mathrm{m}$ thick gold foil. A small, circular, 3D-printed plastic holder both supports the foil and clamps it into the cutout in the cathode. The cutout in the centre of the holder is $1 \mathrm{~cm}$ diameter, and the relief of the holder is $1.5 \mathrm{~mm}$ on each side.

To ensure complete stopping of all fission fragments within the drift region, $\mathrm{SRIM}^{8}$ simulations were performed of typical low-mass, high-energy fission fragments stopping in several candidate gases; from these data, gas pressures were chosen. Three gases were trialed in the commissioning of the detector: $\mathrm{CF}_{4}$, iso- $\mathrm{C}_{4} \mathrm{H}_{10}$, and $\mathrm{P}-10(90 \% \mathrm{Ar}+10 \%$ $\mathrm{CH}_{4}$ ); all run using continuous flow. Out of these, $\mathrm{P}-10$ was found to be the most favourable in terms of pressure and voltage requirements weighed against ion stopping distance, electron drift velocity, and signal height. A pressure of 800 mbar was used.

The grid was connected to ground potential, connected through a $10 \mathrm{M} \Omega$ resistance. The electric field strength in the drift region was chosen based on interpolated data of drift-velocity as a function of reduced electric field strength, taken from the literature; ${ }^{9}$ these data were used to identify regions of electric field strength that would result in stable electron collection times. The field strength in the collection region (CR) was chosen to be several times higher than that in the DR to minimise electron pickup by the Frisch-grid. Electric field strengths used in the current work were $300 \mathrm{~V} / \mathrm{cm}$ and 1200 $\mathrm{V} / \mathrm{cm}$ in the DR and CR, respectively.

Signals from the cathode, grids and anodes were all fed through high-gain integrating pre-amplifiers before being recorded using an $80 \mathrm{MHz}, 14$ bit digital acquisition system. The system had an event window of $6.4 \mu \mathrm{s}$ and was hardware-triggered via a pulse-height discriminator, fed by a shaped copy of the cathode signal.

\section{Data Correction and Analysis}

A suite of DSP algorithms is used to correct and analyse the data collected. The event traces are first treated by a pre-sort program that applies various corrections and records relevant parameters.

Unlike other modern endeavours, ${ }^{10}$ which digitally replicate analogue spectroscopy amplifiers to shape the anode traces, the intention here is to preserve the form of the 
traces so that $\mathrm{dE} / \mathrm{dx}$ can be reconstructed. This relies on high-pass and low-pass IIR (Infinite Impulse Response) filters with sharp cut-offs to isolate out baseline and high frequency noise components without distorting the traces. The fall time of the preamplifiers is corrected as an exponential function of trace height, coefficients for this being obtained from pulser signals recorded prior to fission-data collection. Gain matching between pre-amplifier channels is corrected for, also using previously determined coefficients.

Corrections for GI to both anode and grid traces is required. Grid correction is implemented by:

$$
Q_{G}^{c o r r}(t)=\frac{Q_{G}(t)}{1-G I}
$$

and anode correction by:

$$
\boldsymbol{Q}_{A}^{c o r r}(t)=\boldsymbol{Q}_{A}(\boldsymbol{t})-\left(\frac{\boldsymbol{Q}_{C}(t) G I}{1-G I}\right),
$$

where $\mathrm{Q}_{\mathrm{C}}$ is the cathode reconstruction, ${ }^{11}$ obtained by summation of grid and inverted anode signals.

The GI is derived directly from the fission data as the first order coefficient that fits the grid signal to the summed grid and anode signals by least squares reduction. The value of GI used in the correction is the running average of the centroid of the GI spectra updated every 20,000 events.

After initial corrections, the traces are gated on a number of parameters to remove those subject to pileup, or having otherwise anomalous characteristics. FIR (Finite Impulse Response) filtering is used to smooth the anode and grid traces before the first and second derivatives are taken; cubic interpolation is then used to reduce the width of the time step from $12.5 \mathrm{~ns} /$ channel to $1 \mathrm{~ns} /$ channel. The start-times and end-times of the traces are identified by looking for the half-height time-channels of the peaks in the second derivatives, the side furthest from the centre of the trace; the peak SP is the fullheight of the first derivative; the average heights of the unfiltered anode and grid signals after their end-times are $\mathrm{Q}_{\mathrm{A}}$ and $\mathrm{Q}_{\mathrm{G}}$ respectively.

Values for the anode and grid charges and full timing data are written out in 10,000 event blocks. Start-time of the event is defined to be the mean of the SS and BS grid signal start-times and the beginning of electron collection, the anode start-time. The end of collection is defined as the mean of the grid and anodes signal end-times for each region of the detector. From these data, distributions are constructed to which equations (2) and (3) can be applied, and $\theta$ obtained. This is performed by polynomial fitting to the half-heights of the distributions in each energy channel.

Finally, angular dependent energy and SP losses can be determined and corrected for and mass spectra generated. Three variations of angular determination technique are used: grid method, ${ }^{6}$ drift-time method and collection time method. ${ }^{7}$ Further details are given in Sec. 5. Due to the nature of these techniques, second order corrections to the resulting distributions are necessary. 


\section{Results and Discussion}

The distributions generated from the anode-charge, grid-charge and timing-data from the SS region are presented in Figs. 3a) to c). Results are consistent with those expected: the range of distributions of $\mathrm{Q}_{\mathrm{G}} / \mathrm{Q}_{\mathrm{A}}, \mathrm{T}_{\text {drift }}$ and $\mathrm{T}_{\text {coll }}$, corresponding to the grid method, drift time method and collection time method respectively, all varying as a function of energy. For each distribution two fits are shown: one corresponding to a $0^{\circ}$ emission angle and the other chosen to correspond to $80^{\circ}$ due to the relief of the source holder cutting out very high angle events.



a)

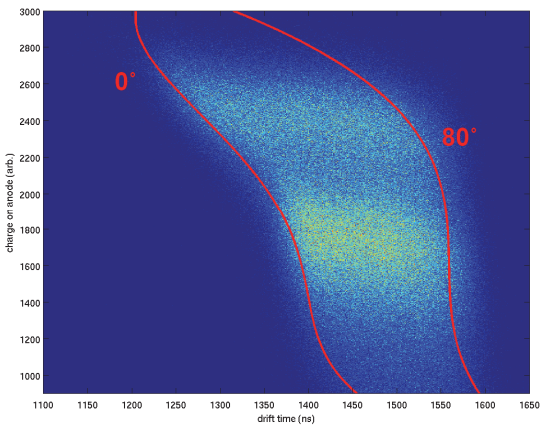

b)

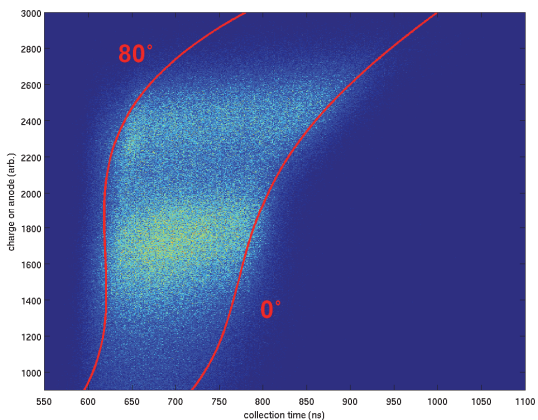

c)

Fig. 3. Two dimensional distributions of angular determination parameters and energy, showing polynomial fits used: a) grid method, energy vs. $\mathrm{Q}_{\mathrm{G}} / \mathrm{Q}_{\mathrm{A}} ; \mathrm{b}$ ) drift time method, energy vs. drift time; c) collection time method, energy vs. collection time.

The angular distributions for both SS and BS regions, resulting from each of the three methods, are presented in Figs. 4a) to c), and a comparison of the difference in angles between each region for each event is shown in Fig. 5. Distributions determined by the grid method showed a bias toward lower angles and the smallest difference between SS and BS, while the collection time method showed a bias toward higher angles and the largest difference between SS and BB. The drift time method shows the flattest angular distribution and a SS BS difference only slightly poorer than the grid method. 


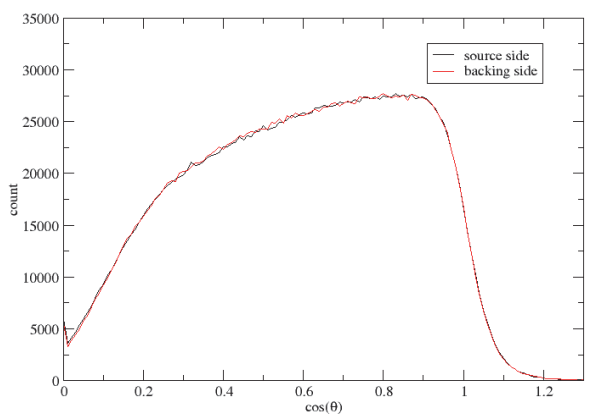

a)

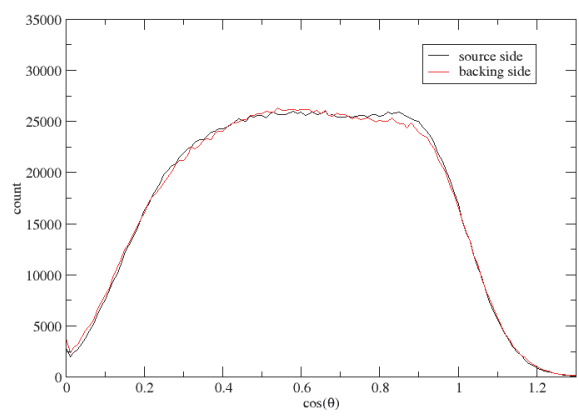

b)

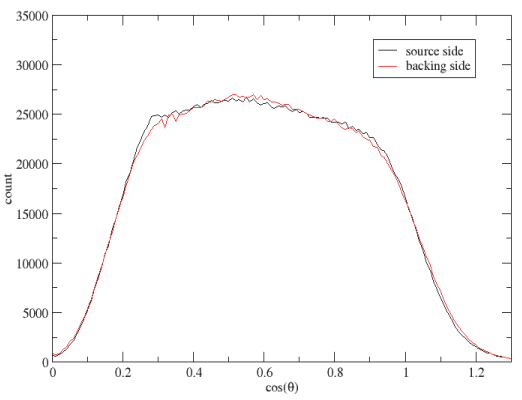

c)

Fig. 4. Angular distributions determined using the grid, drift time and collection time techniques for both SS and BS regions: a) grid method; b) drift time method; c) collection time method.

Previous work $^{7}$ has used the FWHM of the SS BS angular difference peak as a measure of accuracy of the angular determination, but care should be taken when applying this method. Using the results obtained from the grid method in this paper as an example, the skew of the $\cos (\theta)$ distribution indicates a general biasing of the values assigned and this naturally leads to a sharpened difference peak. The cited paper also points to other factors such as increased thickness of, and therefore scattering in, the source and backing, which contribute strongly to widening of the difference peak. The $\mathrm{Au}$ backing used in the present work means that large differences in the angles from each region of the detector are anticipated, and indication of angular resolution from this alone is unreliable.

Figure 6 shows average energy as a function of $1 / \cos (\theta)$. This gives evidence for the requirement of second order corrections, in that the dependency of average energy on angle does not vary linearly as would be expected. ${ }^{7}$ When performing the first order energy corrections, a polynomial fit was therefore used to avoid adding large skew to the resulting energy distribution at higher angles. 


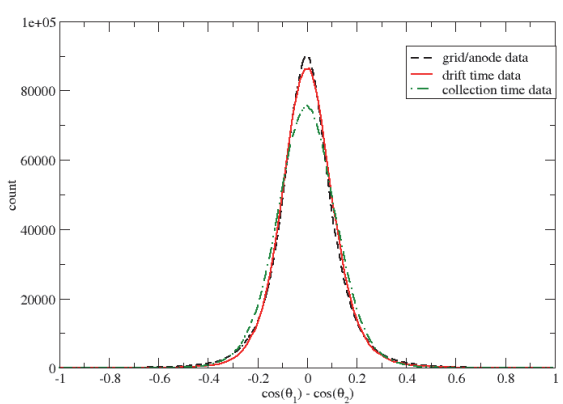

Fig. 5. Difference in $\cos (\theta)$ between SS and BS for three methods of angular determination.

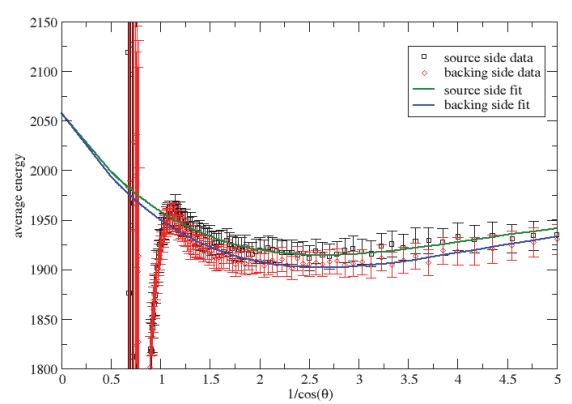

Fig. 6. Average energies as a function of $1 / \cos (\theta)$ with fitted polynomials.

Energy, SP and mass distributions after first order angular determination and angular dependent energy loss correction are presented in Figs. 7-9, respectively. From Fig. 7 (left), it can be seen that SS and BS energies are well matched, and from Fig. 7 (right), that there is no significant dependence of energy on angle, although the need for second order corrections to $\cos (\theta)$ is evident, particularly for high energy fragments at high angles. Figure 8 (left) shows a shift in SPs between the two detector regions, which is attributed to them having a slight difference in drift velocity; while Fig. 8 (right) shows a clear dependence of SP on angles, as is expected. Both these dependencies are to be removed with further correction. The mass distribution presented in Fig. 9 shows two clear peaks with good symmetry, but the valley falls to just 0.17 of the full height. This can be attributed, initially, to the need for second order corrections to energy, but a limit will ultimately be reached where the energy spread from the thick source and backing becomes the dominant source of error. Figure 8 (right) again shows the requirement for second order corrections to $\cos (\theta)$.
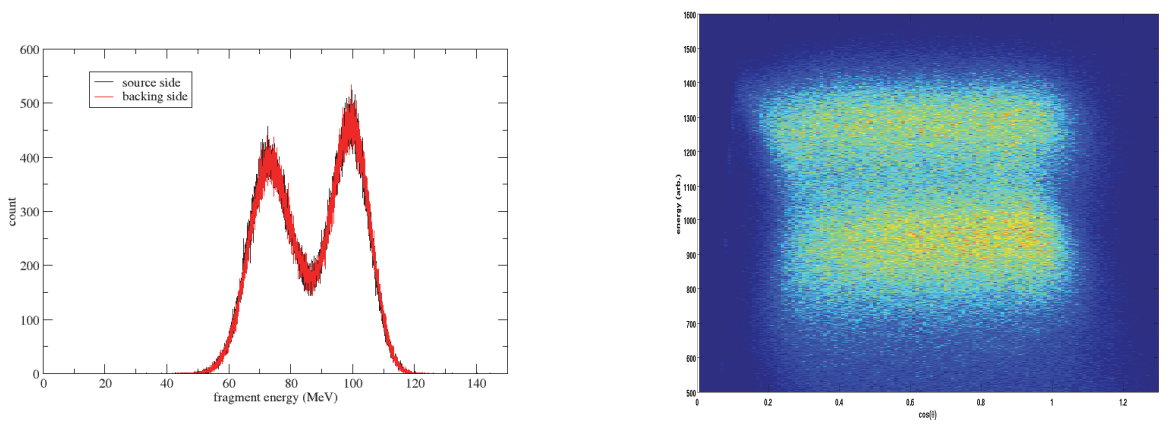

Fig. 7. Left: corrected energy spectra for each region of the detector. Right: energy vs. angle distribution for the SS region. 

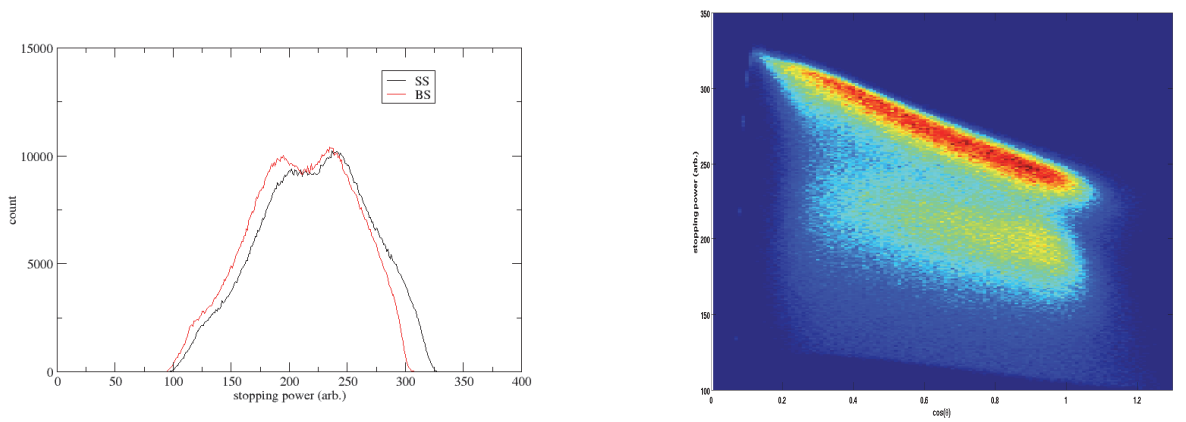

Fig. 8. Left: corrected (for energy related losses) stopping powers for each region of the detector. Right: stopping power vs. angle distribution for SS.
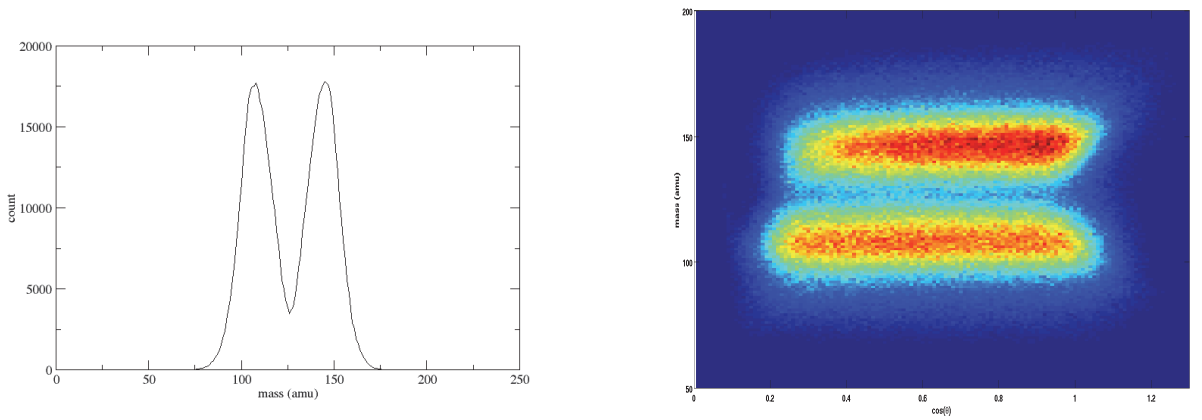

Fig. 9. Left: mass distribution generated form the corrected energies, by the EE method, for SS region of the detector. Right: mass vs. angle distribution for SS.

\section{Conclusions}

A double gridded-Bragg chamber has been designed and commissioned for the study of fission fragment energy, charge, mass and angular distributions. A novel method of grid inefficiency correction has been employed.

The operation of the detector has been verified by application of several of the most modern angular determination and energy correction techniques and by comparison of the results to those found in the literature. Of the three angular determination methods compared and contrasted, it has been found that the drift time measurement produces the most even distribution on single application. It was also found that results improved when multiple methods were used simultaneously, but that regardless of the technique employed, iteration was required.

The detector is now ready to be used as a platform for the development of novel angular determination techniques and ultimately form the basis for the design of a compact detector to be used in conjunction with large HP-Ge detector arrays. 


\section{Acknowledgments}

Many thanks to everyone at both the Nuclear FiRST DTC and the Manchester Nuclear group.

\section{References}

1. F. Gonnenwien, Mass, Charge and Kinetic Energy of Fission Fragments - The Nuclear Fission Process (CRC, 2000).

2. H.W. Schmitt, J.H. Neiler, F.J. Walter, Phys. Rev. 141 3, 1146 (1966).

3. W. Shockley, J. Appl. Phys, 9, 635 (1938).

4. O. Bunemann, T.E. Cranshaw, and J.A. Harvey, Canadian J. Res. 27a, 191 (1949).

5. A. Al-Adili, F.J. Hambsch, R. Bencardino, S. Oberstedt, and S. Pomp, Nucl. Instrum. Meth. A 673, $116(1983)$.

6. A. Al-Adili, F.J. Hambsch, R. Bencardino, S. Pomp, S. Oberstedt, and Sh. Zeynalov, Nucl. Instrum. Meth. A, 671, 103 (2012).

7. A. Gook, M. Chernykh, J. Enders, A. Oberstedt, and S. Oberstedt, Nucl. Instrum. Meth. A 621, $401(2010)$.

8. J.F.Ziegler, J.P.Biersack, M.D.Ziegler, The Stopping and Range of Ions in Matter, $15^{\text {th }}$ edn. (lulu Press Co., 2015).

9. L.G. Christophorou, D.L. McCorkle, D.V. Maxey, and J.G. Carter, Nucl. Instrum. Meth. 163, 141 (1979).

10. O. Zeynalova, Sh. Zeynalov, F.J. Hambsch, S. Oberstedt, and I. Fabry, DSP Algorithms for Fission Fragment and Prompt Fission Neutron Spectroscopy, in AIP Conf. Proc. 1186, 430 (2009), http://dx.doi.org/10.1063/1.3265358.

11. A. Gook, F.J. Hambsch, A. Oberstedt, and S. Oberstedt, Nucl. Instrum. Meth. A 664, 289 (2012). 